\title{
A Community-Based Study of Hypertension and Cardio-Metabolic Syndrome in Semi-urban and Rural Communities in Nigeria
}

\author{
Article by Dr. Orji Ikechukwu Anthony \\ Disease Control Unit, Health Department, AMAC, Abuja, Nigeria. \\ Email: drtony2013@gmail.com
}

\begin{abstract}
This work is a critical review of the article titled "A community-based study of hypertension and cardio-metabolic syndrome in semi-urban and rural communities in Nigeria authored by Ulasi Ifeoma. I., Ijoma Chinwuba. K. \& Onodugo Obinna. D and published in BMC Health Services Research journal of 2010, volume 10, page 71 with DOI number; doi: 10.1186/1472-6963-10-71 which was retrieved from http://www.biomedcentral.com/1472-6963/10/71. This review appraised literature related to the subject, evaluated the article structure, authority, relevance, currency, accuracy, stability, and objectivity. Other sections appraised were the tables, recent advances on the subject, accessibility, and credibility of the article. The reviewed original article has its objective as determining the prevalence of Cardio Metabolic Syndrome in rural \& semi-urban communities in South-East Nigeria, with attention to the general population and population with hypertension. The authors involved the use a cross-sectional design in this population based study which involved adult residents of the selected communities between the ages of 25 \& 65 years. They were screened for metabolic syndrome using standard equipment and interviewed with structured questionnaires. The results yielded prevalence among general population of $18 \%$ and $10 \%$ for Cardio Metabolic Syndrome in the semi-urban \& rural community respectively. While among the group with hypertension, the authors recorded a prevalence of $34.7 \%$ and $24.7 \%$ in the semi-urban and rural communities respectively. The study demonstrated a high prevalence of Cardio Metabolic Syndrome especially as it concerns semi-urban communities as well among the population with hypertension

Fundamentally, this is a current and relevant work in the area of non-communicable disease epidemiology in Africa and can be generalized to Blacks in other parts of the world. The work has contributed significantly to the pool of knowledge and can be referenced in future works as it's also an avenue that can be explored for future collaborations in research regarding this topic. The work was written objectively, clearly presented, easy to understand and accessible for researchers, academicians, clinicians and other players in the health sector and can serve the members of the above-listed groups in their various capacities.
\end{abstract}

Keywords: Cardio Metabolic Syndrome, Hypertension, Prevalence, semi-urban community, Rural Community.

\section{Introduction}

This is a critical review of the research; "A community-based study of hypertension and cardiometabolic syndrome in rural and semi-urban communities in Nigeria' authored by Class Ifeoma. I., Ijoma Chinwuba. K. \& Onodugo Obinna. D and published in the BMC health services research journal. It is a fact that Cardiometabolic syndrome is known to be increasing globally (Eckel et.al, 2005), with evidence that hypertension and its complications is more prevalent in Blacks and Africans (Gibbs et.al, 1999) as well as the reality that many Africans especially those in urban and semi-urban settlements are fast switching to western food and lifestyle. Therefore, a study of this cardiometabolic syndrome is very important and relevant in Nigeria being a developing Nation. In this article review, the appraisal of related literature will first be undertaken; this will be followed by a brief summary of the work. Then, the scrutiny of the effectiveness of the structure of the article, thereby, examining the arrangement of the 
Texila International Journal of Public Health

Volume 5, Issue 3, Sep 2017

information in the context of easy access in the event of reading the work. Furthermore, the article will be critiqued, involving the evaluation of the authority, accuracy, and currency of the article, closely followed by the assessment of its objectivity, relevance as well as its stability. Other aspects of the review will include the analysis of the tables \& figures, appraisal of the recent advances as it concerns the study, evaluating the accessibility and credibility of the work.

\section{Literature review}

Hypertension plays a significant role in the pathophysiology of stroke, coronary heart disease and other cardiovascular complications (Parks, 2013). It is one of the components of the cardiometabolic syndrome as defined by the IDF classification, (IDF, 2006). Hypertension is referred to as diastolic blood pressure of greater than or equal to $90 \mathrm{mmHg}$ and/or systolic blood pressure of $140 \mathrm{mmHg}$ or greater. (WHO. 2003) and is found to be of a high prevalence in sub-Saharan Africa subsequent to the findings of Opie et.al, 2005. According to Guize et.al, (2008), metabolic syndrome can be defined as an association of central obesity with some other cardio-metabolic risk factors especially linked with high risk of sudden death as well as stroke. Obesity, on the other hand, is a medical condition characterized by excess body fat which usually affects the health of the individual adversely in addition to increasing the risk of cardiovascular \& other diseases (Lucas \& Gilles, 2003). In the work of Akpa et, al, 2008, obesity has severe adverse health impact resulting in very poor health outcome in addition to mortality ensuing from cardiovascular diseases. Furthermore, diabetes mellitus which is defined as a random blood sugar of greater than $200 \mathrm{mg} / \mathrm{dl}$ (Isara et.al, 2015), or fasting blood sugar of greater than 126mg/dl (Lucas 7 Gilles, 2003) has been identified as risk factor for coronary heart disease according to the works of Hoogwerf \& Huang, 2012, with a very heavy mortality burden as reported by WHO (2015). It is also an established fact that the risk factors for developing diabetes mellitus are commoner in developing countries (Ibrahim, et, al, 2012).

The metabolic syndrome prevalence has been shown to be on the increase among Africans, this is supported by this article which recorded overall prevalence OF $18 \%$ in semi-urban population in Enugu, Nigeria which is similar to the works of (Tran et. al. 2011) which recorded 17.9\% in Addis-Ababa Ethiopia. Both studies used the IDF definitions of metabolic syndrome (IDF, 2006). The hypertension component of metabolic syndrome among the overall population was $41.1 \%$ in this study but $21.8 \%$ of the male population of the Ethiopian study. The studies concluded that metabolic syndrome and its individual co-morbidities are prevalent among Africans.

\section{Article summary}

The objective focused on the prevalence of Cardio Metabolic Syndrome in rural \& semi-urban communities in South-East Nigeria, with attention to the general population and population with hypertension. The work is a cross-sectional population-based research whose study population was adults aged 25 years up to 64 years selected from the target rural and semi-urban community in the study area. Using qualified medical personnel, participants were screened for Cardiometabolic Syndrome by the IDF criteria (IDF 2006), the anthropometric parameters for the Europeans were employed while for hypertension, the criteria of WHO \& ISH (WHO/ISH, 2003) was employed. A structured questionnaire was used for the interview while the weight, height, hip and waist circumference were measured with a standardized weighing scale, stadiometer and me asuring tape respectively. A semi-auto-analyzer Photometer was used to measure the lipids while Accuchek glucometer was used to measure the blood glucose level. The findings of the study revealed a prevalence of $18 \%$ for Cardio Metabolic Syndrome in the semi-urban settlement and $10 \%$ in a rural community as found in the general population, thus showing the prevalence semi-urban communities to be almost twice as that found in rural communities. However, among the group with hypertension, a much higher prevalence of $34.7 \%$ and $24.7 \%$ were recorded in the semi-urban and rural communities respectively. Overall, a high prevalence of Cardio Metabolic Syndrome was demonstrated in the study especially as it concerns semi-urban communities as well among the population with hypertension 


\section{Article structure}

The authors introduced the article with an abstract which briefly presented the background of the study, the justification and objective as well as the methods and overview of the findings of the research. The main body of the text is sub-divided into various sections which enhanced the easy accessibility of the information from the work. The following main sections are clearly outlined: background, methods, results, discussions and conclusion. There are also sub-sections within methods and result sections as well as other addendums like competing interest declaration, authors' contributions, acknowledgment, and references sections. The background clearly described the problem backed up with the review of related literature, an objective was stated and the need for the study was justified. The method was broken down into sub-sections describing the study area, population, sample size, sample design, sample selection, study personnel, data tool, ethical review, clinical evaluation and collection of blood sample: the information were unambiguously presented thereby improving the quality of the work. The parameters under study were defined and the criteria used stated with links given in the definition sub-section while a description of the statistical analysis was also done. The results and discussion sections were subdivided into subsections, addressing the various variables being studied, presenting the findings and discussions. These were illustrated with tables and figure and the strategy help the reader to understand the information and easily access them. Moreover, the discussion was enriched with relevant citations supporting the findings. The limitations of the study were properly stated which can guide future researchers in the area they can improve. The conclusion was shallow, needed to be enriched though it was able to bring out the substance in the findings of the research. Largely, the structure of the article is logically developed.

\section{Critique}

\section{Authority}

BMC Health Services Research is a reputable peer review journal with a track record of unbiased objective publications. The work was retrieved from Google Scholar search, a scholarly article search engine and equally reputable. The authors are seasoned researchers and academicians with several years of research and teaching experience in the field of internal medicine as well as clinical experience managing these non-communicable diseases in one of Nigeria's foremost University, University of Nigeria Teaching Hospital in Enugu. These are the authority in the field of non-communicable disease management and have authored several other articles in this field. Furthermore, the combination of their individual experiences as equal contributors in this work speaks volume of the quality and authority of the work.

\section{Accuracy}

The article is a product of a current and recent research study giving information that is accurate as at this period. This is supported by a bibliography list of current and reliable research works which were cited in the main text giving credence to the research work and enriched the literature review. Furthermore, the synergy of the three authors at different stages of drafting and revising the work, as well as the role of the peer review journal which takes on a rigorous review process in editing works before publication in addition to other credible sources that the work links to, all hold up to the accuracy of the article.

\section{Currency}

The research from which this article under review is published describes a recent work which was carried out in 2009 and submitted same year for publication. Following months of a rigorous review process, the work was accepted and published in March 2010 which upholds its currency. Moreover, the article cited recent and current references in the main text which were articles published between 1997 and 2009 except for only one article which was published in 1983. Taken as a whole, the article is a 
Texila International Journal of Public Health

Volume 5, Issue 3, Sep 2017

current work in view of the date of execution of the research, its publication and the recent references it parades.

\section{Relevance}

This article was published in a scholarly research journal and will be very relevant to the research, health professionals, and academic communities. It was written to inform the clinicians, the academicians, the researchers and most of all, to enlighten the policy makers and inform them for decision making as regards policy for these non-communicable diseases. It can also serve as a guide to Health Organization interested in Non-Communicable disease control to understand the extent of the burden of these conditions in our environment in addition to serving as a wake-up call to other civil society organizations to face up the challenge and join the fight to stem the rising tide of non-communicable diseases in our environment.

\section{Objectivity}

This research work was objectively developed and systematically conducted. The design was superb and the process rigorous, yet painstakingly followed. For instance, the use of the WHO STEPS (WHO, 2006) criteria for calculating the sample size was followed to the letter. The participants for the study were stated: adults aged 25-64 years of age selected from the target community. Moreover, the use of The WHO STEPS and KHDC (KHDC, 2005) questionnaire for such studies as a template for developing the questionnaire for the study was very objective. The ethical approval for such population-based study was duly obtained. Additionally, the definitions of the Cardiometabolic Syndrome diagnosis using the criteria for Europids was clearly stated and the component measurement cut off points were outlined clearly making it easy for the reader to appreciate the process.

\section{Stability}

This research work being an article published incredible peer-review journal is a stable resource material. This is a health services research journal relevant for academic, health intervention and researchers reference.

\section{Analysis of graph/Chart/Figure/Table}

Table 1: Demographic characteristics of the study population

This relevant table showed demographics which explain the findings, for example, the mean age of participants with Cardio Metabolic Symptoms was found to be higher than those without the condition.

Table 2: Anthropometric characteristics, physical activity and prevalence of CMS and Hypertension in study population

The table clearly reported the prevalence of hypertension as well as the prevalence of Cardio Metabolic Syndrome as related to the objective.

Table 3: Prevalence of co-morbidities in non-hypertensive, hypertensive and general population in the various communities

This table is relevant to ascertain the risk factors, associated co-morbidities, and protective factors.

Table 4: Prevalence of population health determinants in general and hypertensive populations in the communities

The table is relevant as it showed the association between the population health determinants and Cardio Metabolic Syndrome cum hypertension.

Figure 1: Prevalence of hypertension by number of components of MS

The figure showed that hypertension prevalence increased with the number of Metabolic Syndrome risk factors.

\section{Recent advances related to the topic}


One recent advance in metabolic syndrome concerns the new definitions of the syndrome by some credible experts in this field of medicine.

Following the 2001 definition by (NCEP-ATP III) National Cholesterol Education Program -Adult Treatment Panel III, (see appendix), the American Heart Association (AHA) \& National Heart, Lung, and Blood Institute (NHLBI) created a new definition of the syndrome in 2005. In this definition (see appendix), two changes were made: 1 . the waist circumference was lowered slightly to a value equal to or greater than $102 \mathrm{~cm}$ for men and $88 \mathrm{~cm}$ for women, unlike the NCEP-ATP III which was greater than the respective values for both men and women. 2 . The blood glucose criteria were lowered from equal to or greater than $1.10 \mathrm{~g} / \mathrm{l}$ to equal to or greater than $1.00 \mathrm{~g} / \mathrm{dl}$. Using this criterion, any of the three components are required to make a diagnosis of metabolic syndrome.

Furthermore, the International Diabetes Federation (IDF) also developed their definition (see appendix) in the same year 2005, modifying the NCEP-ATP III as follows: 1. Unlike AHA/NHLBI, the waist circumference was reduced to equal or greater than $94 \mathrm{~cm}$ in men and equal or greater than $80 \mathrm{~cm}$ in women. 2. As in AHA/NHLBI criteria, the blood glucose was lowered to equal to or greater than 1.00 $\mathrm{g} / \mathrm{dl}$, but added 'or individual with diabetes'. Besides, in contrast to AHA/NHLBI, it also included individuals receiving treatment for lipid abnormalities and hypertension and in addition, requires abdominal obesity plus any other two components for diagnosis.

Moreover, European Society of Cardiology (ESC) and European Society of Hypertension (ESH) reviewed metabolic syndrome definition in 2007 and came up with an international consensus that it is a specific association of any three of the components contained in the three previous definitions by NCEPATP III, AHA/NHLBI and IDF. The ESC/ESH definition combined the criteria of NCEP-ATP III as regards blood pressure, triglyceride and abdominal obesity, however, it introduced new threshold for female HDL-C level (less than $0.46 \mathrm{~g} / \mathrm{L}$ ) and for fasting glucose (greater than or equal to $1.0 \mathrm{~g} / \mathrm{L}$ ) similar to AHA/NHLBI and IDF criteria. (Adapted from Guide et, al, 2008).

\section{Conclusion}

The article, 'A community-based study of hypertension and cardio-metabolic syndrome in semi-urban and rural communities in Nigeria' has been thoroughly reviewed and the contents, structure, strength and weakness evaluated and critiqued. Generally, this is current and relevant work in the area of noncommunicable disease epidemiology in Africans and can be generalized to Blacks in other parts of the world. The work has contributed significantly to the pool of knowledge and can be referenced in future works as it's also an avenue that can be explored for future collaborations in research regarding this topic. The work is written objectively, clearly presented, easy to understand and accessible for researchers, academicians, clinicians and other players in the health sector and can serve the members of the abovelisted groups in their various capacities. Cardio Metabolic Syndrome is on the increase in developing countries, fast becoming an epidemic especially among populations with hypertension, thus, it requires a pragmatic step to stem this tide. A holistic approach involving health education for lifestyle changes targeting the masses, clinicians sensitization for patients screening and adequate treatment as well as Organization and Government for policy change to improve funding for intervention to control noncommunicable diseases especially Cardio Metabolic Syndrome.

\section{References}

[1]. Akpa, M.R. \& Mato, C.N. (2008). Obesity in Nigeria: Current trends and management. Nigerian Medical Practitioner. 54 (1), 11-15. Retrieved from http://www.ajol.info/index.php/nmp/article/view/28941

[2]. Eckel, R.H., Grundy, S. \& Zimmet, P. (2005). The metabolic syndrome. Lancet 2005, 365, 1415-1428. Retrieved from http://www.biomedcentral.com/pubmed/15836891.

[3]. Gibbs, C., Beever, D. \& Lip, G. (1999). The management of hypertensive diseases in black patients. QJM 1999, 92:187. Retrieved from http://www.biomedcentral.com/pubmed/10399605 
Texila International Journal of Public Health

Volume 5, Issue 3, Sep 2017

[4]. Guize, L., Pannier, B., Thomas, F., Bean, K., Jégo, B. \& Benetos, A., (2008). Recent advances in metabolic syndrome and cardiovascular disease. NCBI resources 101(9), 577-583. Retrieved from www.ncbi.nlm.nih.gov/pubmed/19041842. doi:10.1016/j.acvd.2008.06.011

[5]. Hoogwerf, B. J., Huang. J., C. Cardiovascular Disease Prevention. Cleveland clinic center for continuing education. September 2012. Retrieved from www.cleveland clinicmeded.com/cardiovascular disease convention

[6]. Ibrahim M.M. \& Damasceno A. (2012). Hypertension in developing Countries. The Lancet. 380 (9841), 611619.

[7]. International Diabetes Federation, (2006). The IDF consensus worldwide definition of the metabolic syndrome. Retrieved from http://www.idf.org/webdata/docs/IDF_Meta_def_final.pdf

[8]. International Society of Nephrology, (2005). Program for detection and management of chronic kidney disease, hypertension, diabetes and cardiovascular disease in developing countries (KHDC PROGRAM). Brussels, International Society of Nephrology; 2005:17-19.

[9]. Lucas A.O \& Gilles H.M. (2003). Short Textbook of Public Health Medicine for the Tropics, $4^{\text {th }}$ Ed., London, Hodder Arnold. 236-237.

[10]. Opie L.H. \& Seedat. Y.K. (2005). Hypertension in Sub-Saharan African Populations. American Heart Association Journal. 112, 3562-3568. Doi: 10.1161/CIRCULATIONAHA218. Retrieved from http://www.njcponline.com/article.asp

[11]. Park, K. (2013). Parks textbook of Preventive and Social Medicine, $21^{\text {st }}$ Ed, Jabalpur, India. Bhanot. 344-346.

[12]. Tran, A., Gelaye, B., Girma, B., Lemma, S., Berhane, Y., Bekele, T. ,.. Williams, M.A. (2011). Prevalence of Metabolic Syndrome among Working Adults in Ethiopia. SAGE-Hindawi access to research international journal of hypertension. 2011, Article ID 193719, 8 pages doi:10.4061/2011/193719

[13]. World Health Organization: International Society of Hypertension Writing Group (2003). World Health Organization/International Society of Hypertension (ISH) statement on management of hypertension. Journal of Hypertension, 2003, 21:1983-1992. Retrieved from http://www.biomedcentral.com/pubmed/14597836

[14]. World Health Organization, WHO. (2003). International Society of Hypertension Writing Group. World Health Organization/International Society of Hypertension (ISH) statement on management of hypertension. Journal of Hypertension, 21, 1983-1999.

[15]. World Health Organization STEPS Manual, (2006). Retrieved from http://www.who.int/chp/steps/manual/en/index.html

[16]. World Health Organization, (2015). Diabetes Fact sheet. Retrieved from http://www.who.int/mediacentre/factsheets/fs312/en/ 